\title{
ВИДЫ НЕЙРОСЕТЕВЫХ ТЕХНОЛОГИЙ, ПРИМЕНЯЕМЫХ В ЭКОНОМИКЕ
}

\section{METHODOLOGY FOR EVALUATING THE EFFECTIVENESS OF A COMMERCIAL ORGANIZATION}

\section{E. Cozac}

Summary. Modern conditions of the digital economy are characterized by a large flow of information data coming from numerous sources that human intelligence is not able to process. The reason for this is not only a sufficiently large amount of data, but also their uncertainty, as well as not infrequently their insufficiency. The resulting problem of effective pattern recognition, necessary for analysis and forecasting in the economy, began to be solved by using neural networks in mathematical programming.

Over the past few years, there has been a significant increase in the use of artificial intelligence capabilities in the economy as a result of the development of neural network technology. The creation of new algorithms based on the fundamental principles of the work of the ordinary human brain made it possible to process much more economic information. Due to the fact that neural networks are not programmed, but are independently trained using the analysis of previous experience, their use allows to reduce the percentage of errors, increasing the efficiency of economic entities.

The article discusses the types of neural networks that are currently used in the economy in order to solve various problems. Six classification features of such neural networks are identified. In recent years, convolutional neural networks, which have great capabilities compared to other types of neural network models, have become the most widespread in the economy.

Keywords: neural network technologies, neural networks, pattern recognition, digital technologies.

\author{
Козак Евгений \\ Cтарший разработчик, Memery Crystal LLP, Лондон, \\ Англия \\ eugeniu.cozac@gmail.com
}

Аннотация. Современные условия цифровой экономики характеризуются большим потоком информационных данных, поступающим из многочисленных источников, что не способен обработать человеческий интеллект. Причиной этому является не только достаточно большой объем данных, но и их неопределенность, а также не редко их недостаточность. Возникшая в результате этого проблема эффективного распознавания образов, необходимого для анализа и прогнозирования в экономике, стала решаться при помощи использования нейронных сетей в математическом программировании.

За последние несколько лет произошел значительный рост в области использования возможностей искусственного интеллекта в экономике в результате развития технологии нейронных сетей. Создание новых алгоритм0в, построенных на базе принципиальных основ работы обычного человеческого мозга, дали возможность обработки намного большей экономической информации. В связи с тем, что нейронные сети не программируются, а самостоятельно обучаются с помощью анализа предыдущего опыта, их использование позволяет снижать процент ошибок, повышая эффективность деятельности экономических субъектов.

В статье рассматриваются виды нейронных сетей, которые в настоящее время применяются в экономике с целью решения разнообразных задач. Выделено шесть классификационных признаков таких нейронных сетей. В последние годы наибольшее распространение в экономике получили сверточные нейронные сети, обладающие большими возможностями по сравнению с другими видами моделей нейросетей.

Ключевые слова: нейросетевые технологии, нейронные сети, распознавание образов, цифровые технологии.

Понятие «нейронная сеть» было сформулировано достаточно давно, представляя собой алгоритм для машинного обучения. В частности, исследования искусственных нейронных сетей ведут уже не менее 70 лет $[11$, c. 17].

Однако их применение в экономике стало возможным и экономически выгодным только с 2010 года, когда была создана большая база ImageNet, послужившая 

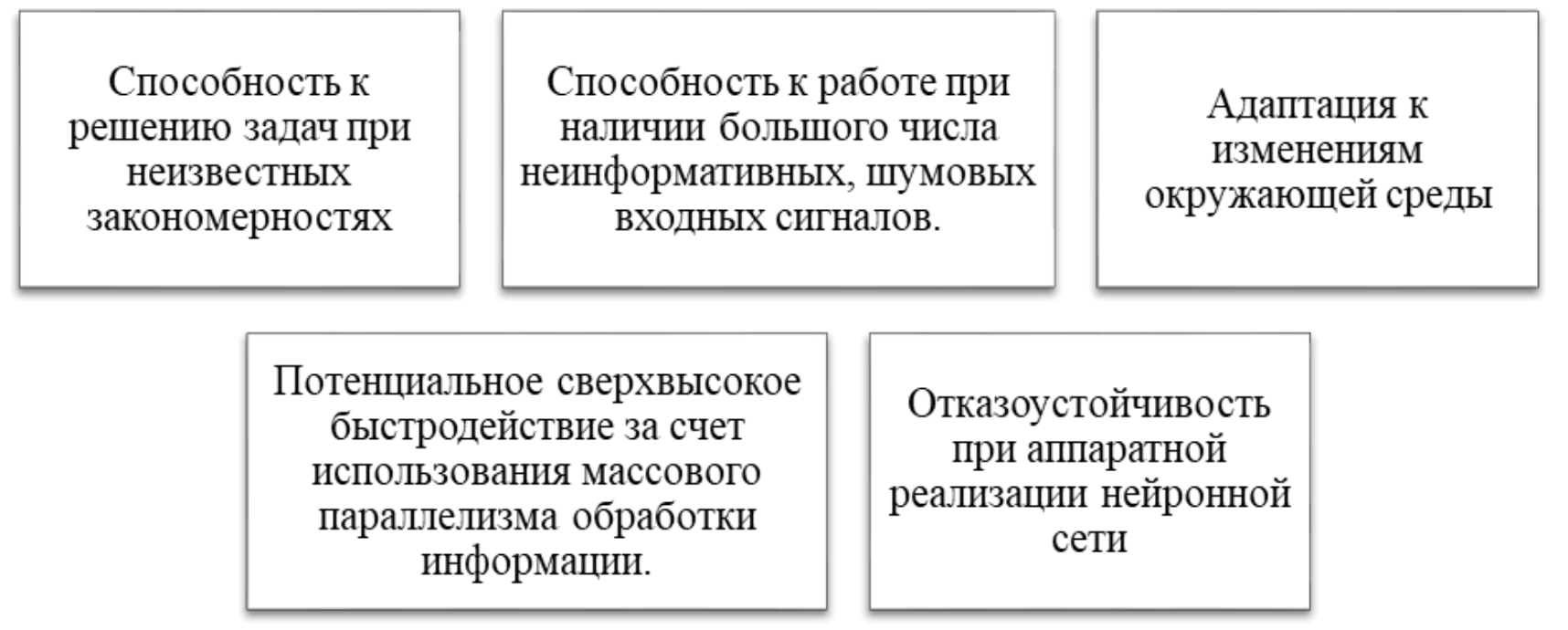

Рис. 1. Преимущества нейронных сетей для использования в экономике Составлено автором по данным $[3,5]$

подходящей выборкой для обучения нейронных сетей. В 2012 году появилась технология глубокого обучения нейронных сетей с ускорением на GPU (графический процессор), что позволило более эффективно их обучать без использования программного обеспечения, написанного программистами [6]. После этого произошел большой шаг вперед в области применения нейронных сетей в экономике.

В настоящее время технологии нейронных сетей находят применение почти во всех сферах жизнедеятельности человека, в том числе в обработке экономической информации и ее прогнозировании.

Основное назначение технологий нейронных сетей заключается в решении нестандартных задач, в которых не созданы алгоритмы решений по причине неполноты или противоречивости входных данных. То есть их применение оправдано, когда не существует явных взаимосвязей и зависимости между информацией на входе и информацией на выходе, что не позволяет решить их математическими или экспертными методами.

На рисунке 1 представлены преимущества нейронных сетей для использования в экономике.

В настоящее время в экономике технологии нейронных сетей применяются разнообразными финансовыми организациями (кредитными организациями, биржами и т.д.), страховыми компаниями, промышленными и государственными компаниями.

Технология нейронных сетей используется почти во всех сферах экономики, где применяется видео- наблюдение. Наряду с другими методами машинного распознавания, нейросетевые технологии созданы для вычленения из большого потока объектов необходимой информации [6].

В современной цифровой экономике нейронные сети используются для решения следующих задач [1, 4]:

- прогнозирование рынков и основных микрои макроэкономических тенденций;

- оптимизация логистических и товарно-денежных потоков;

- оптимизация и совершенствование производственных процессов;

- обработка и анализ разнообразных социальных опросов в целях выявления тенденция в экономике;

- диагностика качества произведенной продукции;

- прогнозирование результатов и динамики политических и экономических рейтингов;

- другие области экономических исследований;

- раскрытие фальсификаций;

- анализ и прогнозирование риска.

При этом эффективность от применения нейронных сетей в прогнозировании и экономических исследованиях постоянно возрастает.

Примеры использования нейронных сетей в различных отраслях экономики представлены в таблице.

Современные поисковые алгоритмы, онлайн-переводчики, сервисы товарных рекомендаций основаны на нейронных сетях. 
Таблица 1. Некоторые примеры использования нейронных сетей в различных отраслях экономики

\begin{tabular}{|c|c|}
\hline Отрасль & Пример применения нейронных сетей \\
\hline Розничная торговля & $\begin{array}{l}\text { Сбор различной информации о количестве и качестве покупателей, времени обслуживания, } \\
\text { правильности расстановки товаров, о мошенничестве продавцов или покупателей, контроль } \\
\text { поведения продавцов при появлении покупателя и другое }\end{array}$ \\
\hline Транспортная отрасль & $\begin{array}{l}\text { Анализ и оптимизация транспортных потоков, логистических сетей, загруженности дорог, } \\
\text { соблюдения правил парковки, сбор информации о пассажирах }\end{array}$ \\
\hline Банковская отрасль & $\begin{array}{l}\text { Сбор различной информации о количестве и качестве клиентов, времени обслуживания, } \\
\text { мошенничестве работников или клиентов, оценка рисков заемщиков, контроль работы } \\
\text { банкоматов и их безопасности, контроль безопасности клиентов }\end{array}$ \\
\hline Общественная безопасность & $\begin{array}{l}\text { Определение нетипичных поз человека, нахождение и распознавание оставленных предметов, } \\
\text { поиск похожих объектов, обнаружение и распознавание лиц и номеров авто, местоположения } \\
\text { людей и других объектов, обнаружение огня, дыма, воды в неположенных местах }\end{array}$ \\
\hline Производство, строительство & $\begin{array}{l}\text { Обнаружение нахождения людей в особо опасных зонах в условиях большого количества } \\
\text { визуальных помех (наличие работающей техники или плохих погодных условий), контроль } \\
\text { за соблюдением правил безопасности на производстве и строительной площадке, } \\
\text { использования спецодежды, контроль технологий и качества изделий / объектов. }\end{array}$ \\
\hline
\end{tabular}

Составлено автором по данным $[2,7,9,11]$.

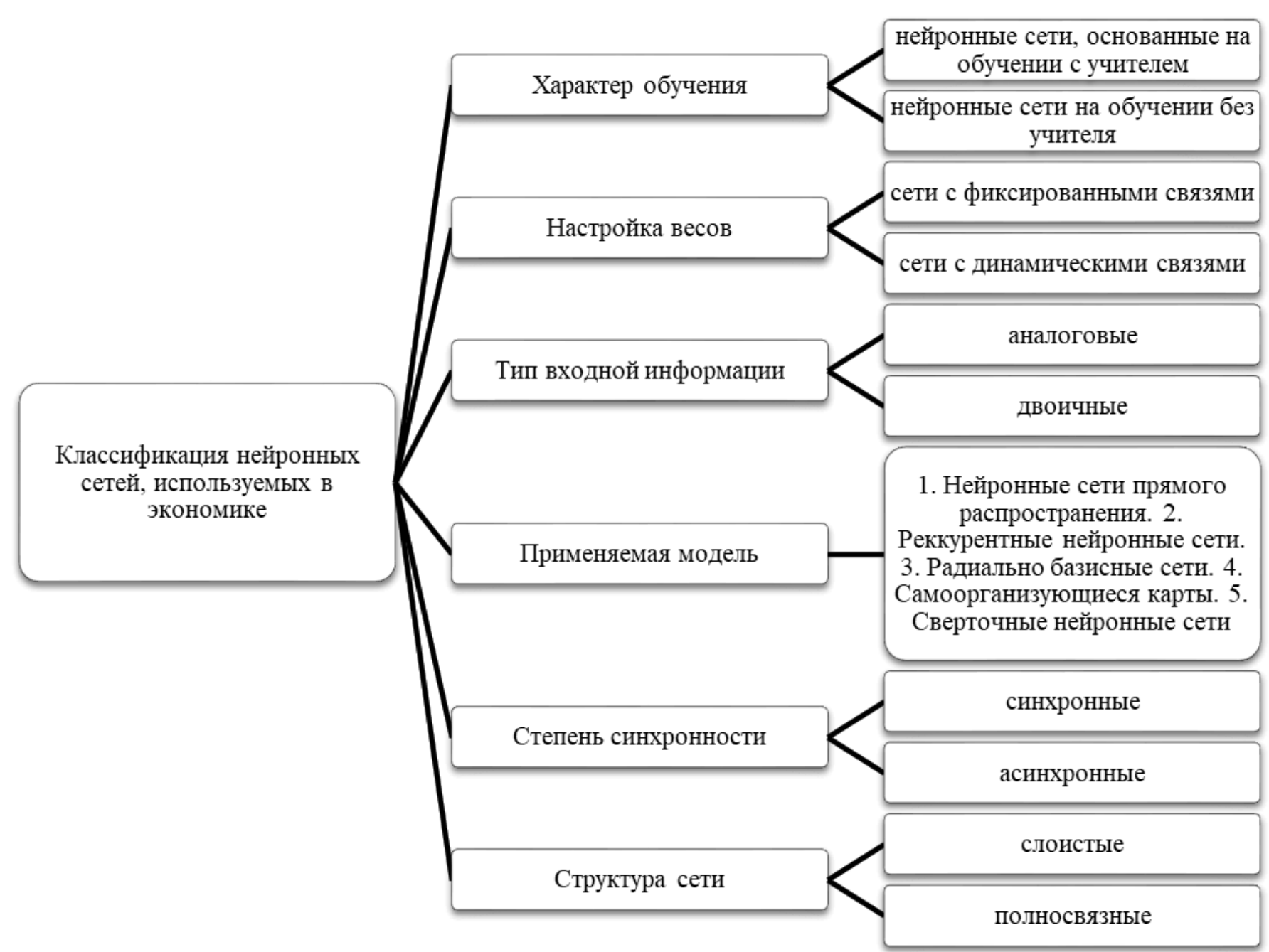

Рис. 2. Классификация нейронных сетей, используемых в экономике Составлено автором по данным $[1,3,5,8]$. 
Далее необходимо рассмотреть виды нейросетевых технологий, используемых в экономике. Их можно классифицировать следующим образом, представленным на рисунке 2.

Согласно представленной классификация нейронных сетей, по признаку «характер обучения» выделяют нейронные сети, основанные на обучении с учителем и нейронные сети на обучении без учителя. Обучение с учителем означает, что в нейронной сети создаются обучающие пары, которые состоят из входного параметра и целевого (конечного) параметра к нему. Далее происходит изменение весов в соответствии с алгоритмом, который направлен на минимизацию ошибок. Обучение без учителя предполагает наличие только входного вектора, при этом выделяют статистические свойства в обучающем множестве, группируя похожие векторы в определенные группы.

Признак «настройка весов» предполагает разделение нейронных сетей на сети с фиксированными связями, когда весовые коэффициенты устанавливаются на начальном этапе по условиям задачи, а также на сети с динамическими связями, когда осуществляется настройка синаптических весов уже в процессе обучения.

Согласно признаку «тип входной информации» выделяют аналоговые нейронные сети, когда информация на входе дается в виде действительных чисел, а также двоичные нейронные сети, когда информация на входе дается в форме нулей и единиц [5].

В соответствии с применяемой моделью нейронной сети выделяют следующие их типы:

1. Нейронные сети прямого распространения, в которых все нейронные связи четко направлены от входных векторов к выходным. Они не имеют петель, что означает, что они не ссылаются на самих себя.

2. Реккурентные нейронные сети, в которых существуют обратные связи, то есть сигнал от нейронов на выходе может частично возвращаться на входные нейроны. Реккурентные нейронные сети организованы так, что каждый из нейронов может получить информацию на входе, как от самого себя, так и от внешней среды [1, с. 308].

3. Радиально базисные сети, которые имеют скрытые слои, состоящие из радиальных элементов, а также выходной слой, состоящий из линейных элементов.

4. Самоорганизующиеся карты или Сети Кохонена, которые обучаются без учителя и эффективно используются в задачах различного распознавания. Сеть Кохонена включает радикальные элементы, как на входе, так и на выходе. Особен- ностью данных сетей является их способность выявлять новизну данных на входе [5].

5. Сверточные нейронные сети, которые дают возможность обеспечения некоторой устойчивости к изменениям размеров, перемещениям, разворотам, изменениям ракурса и другим искажениям, имеющимся в изображениях на входе. Сверточные нейронные сети способны к самостоятельному поиску наилучших признаков для сравнения и группировки данных для более эффективного решения поставленной задачи. Большинство современных моделей нейронных сетей представляют собой сверточные нейросети.

По степени синхронности выделяют синхронные и асинхронные нейронные сети. Их отличие состоит В изменениях состояния нейронов в конкретный момент времени. Так, в синхронных сетях в один момент происходит изменение только одного нейрона, тогда как в асинхронных - нескольких нейронов, составляющих группу или целый слой [8, с. 75].

Исходя из структуры нейронных сетей, существуют слоистые и полносвязные сети. В слоистых сетях нейроны структурированы по группам (слоям), по которым осуществляется обработка информации. Они бывают однослойными и многослойными. В полносвязных сетях каждый из нейронов подает собственный выходной сигнал, как другим нейронам, так и себе. Все сигналы на входе осуществляются всем нейронам [3, с. 139].

Тем не менее, несмотря на разнообразие применяемых в экономике нейронных сетей, они имеют некоторые недостатки. Прежде всего, это их неспособность предоставлять точные и однозначные ответы в трудных условиях распознавания.

Кроме того, к недостаткам также относится многошаговое принятие решений, поставленная задача не решается в один шаг, что замедляет процесс работы. Искусственные нейросети не умеют решать вычислительные математические задачи. Во-первых, сложно донести до сети, какая из частей выражения, например, имеет корень. Во-вторых, отсутствует возможность последовательного решения задачи. В-третьих, существует также проблема невозможности выдачи точных результатов [3, с. 140].

Таким образом, нейронные сети нашли широкое применение в экономике, начиная от распознавания лиц и объектов на фото и видео изображениях, заканчивая оценкой рисков и прогнозированием социально-экономических процессов. При этом эффективность от применения нейронных сетей в прогнозировании 
и экономических исследованиях постоянно возрастает. Существует несколько классификационных признаков нейронных сетей, позволяющих распределить их многообразие на определённые группы. В последние годы наибольшее распространение в экономике получили сверточные нейронные сети, обладающие большими возможностями по сравнению с другими видами моделей нейросетей.

\section{ЛИТЕРАТУРА}

1. Гареева, Г.А. Применение нейронных сетей в экономике / Г.А. Гареева, Д.Р. Григорьева, Т.В. Гилязеев // Молодой ученый. 一 2018. — № 18 (204). C. 306-309.

2. Журавлева, Л.В. Исследования особенностей развития нейронных сетей в современном мире / Л.В. Журавлева, К.А. Стригулин.- Текст: непосредственный // Технические науки: проблемы и перспективы: материалы IV Междунар. науч. конф. (г. Санкт-Петербург, июль 2016 г.).— Санкт-Петербург: Свое издательство, 2016.- С. 9-11.

3. Зенин, А.В. Исследование возможностей использования нейронных сетей / А.В. Зенин // Молодой ученый. — 2017. — № 16 (150). — С. 130-140.

4. Конюхова, О.В., Лапочкина К.С. Применение нейронных сетей в экономике и актуальность их использования при составлении краткосрочного прогноза бюджета // Информационные ресурсы, системы и технологии. - 2012.— № 1. - C. 16-21.

5. Нейронные сети // Портал искусственного интеллекта Aiportal._ URL: http://www.aiportal.ru/articles/neural-networks (дата 0бращения: 26.10.2021)

6. Нейронные сети: новый прорыв. Мнения экспертов // Системы безопасности. — URL: http://lib.secuteck.ru/articles2/all-over-ip/neyronnye-seti-novyyproryv-mneniya-ekspertov/ (дата обращения: 26.10.2021)

7. Нейронные сети // Системы безопасности. — URL: https://www.secuteck.ru/pp-nejronnye-seti (дата обращения: 26.10.2021)

8. Рутковская, Д. Нейронные сети, генетические алгоритмы и нечеткие системы / Д. Рутковская, М. Пилиньский, Л. Рутковский. — М.: РиС, 2013. — 384 с.

9. Степанов, П.П. Искусственные нейронные сети / П.П. Степанов // Молодой ученый. — 2017. — № 4 (138). — С. 185-187.

10. Трофимова, Е.А. Нейронные сети в прикладной экономике: учеб. пособие / Е.А. Трофимова, В.Д. Мазуров, Д.В. Гилёв; под общ. ред. Е.А. Трофимовой. - Екатеринбург: Изд-во Урал. ун-та, 2017. - 96 с.

11. Stanley, K.0., Miikkulainen R. Evolving Neural Networks through Augmenting Topologies, 2002. - P. 14-25.

(c) Козак Евгений ( eugeniu.cozac@gmail.com ).

Журнал «Современная наука: актуальные проблемы теории и практики»

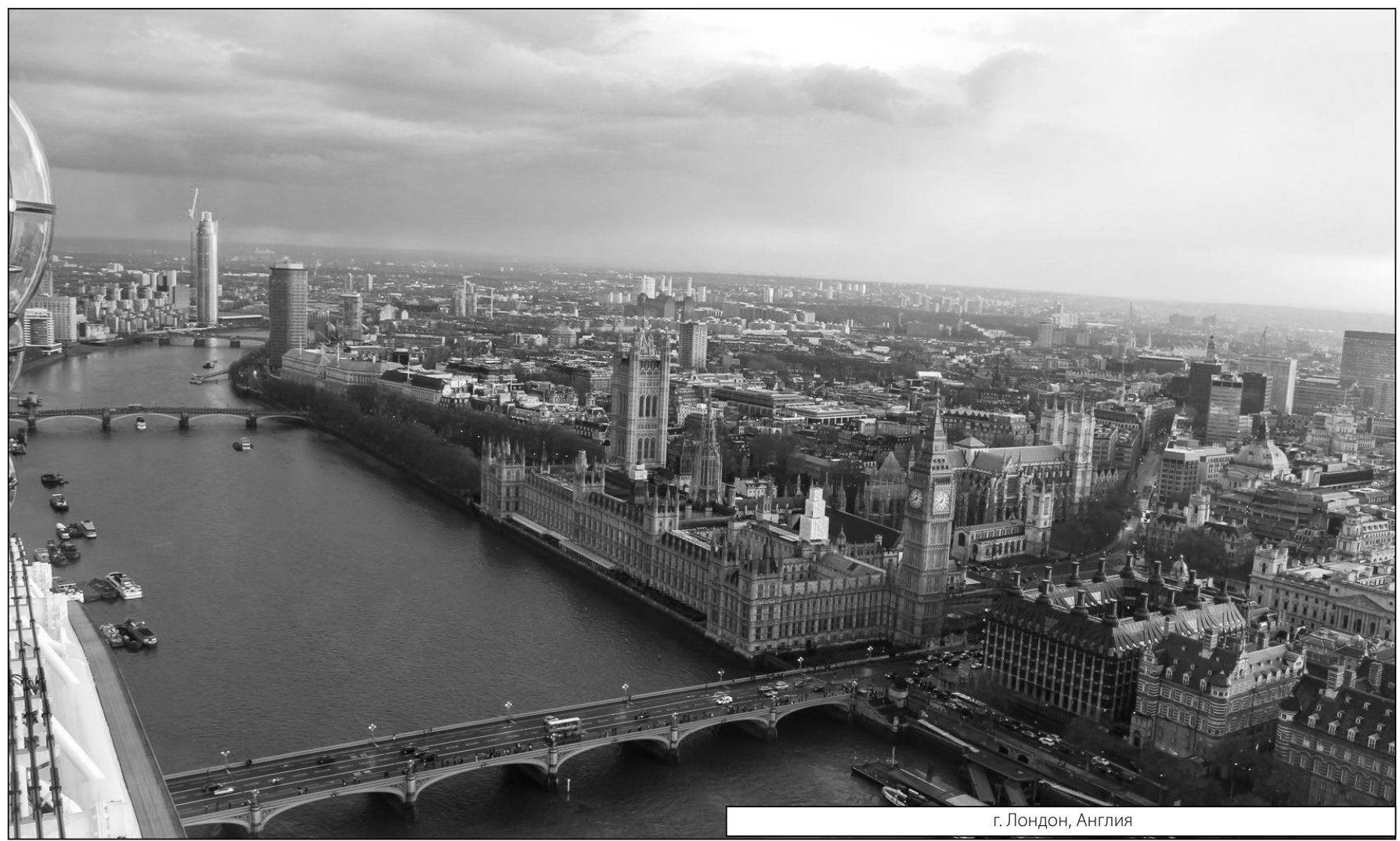

